\title{
Umsetzung von EU-Richtlinien in vier Ländern
}

\section{Hampel, Anja: Musterknaben und Nachzügler. EU-Richtlinienimplementation und nationale Vetospielerkonstellationen im europäischen Vergleich, Tectum Verlag, Marburg 2007, 163 Sei- ten, $€ 24,90$.}

Die Frage der internen Steuerungsfähigkeit der europäischen Institutionen zählt nach der EU-Erweiterung ohne Zweifel zu den spannendsten, dringendsten und zugleich komplexesten Herausforderungen der Europäischen Union. Umso erfreulicher ist es, dass ein wichtiges Instrument für diese Steuerungsfähigkeit - der Einsatz von Richtlinien als Rechtsetzungsinstrument der EU - unter einem wesentlichen Aspekt beleuchtet wird: ihrer zeitgerechten Umsetzung. Der Zeitfaktor scheint sowohl für die Integrationsentwicklung insgesamt als auch für die Bürger innerhalb der EU kritisch zu sein, denn die Mitgliedstaaten nutzen den Freiraum bei der Richtlinienimplementation - gerade bei umstrittener Materie - allzu häufig, um die Umsetzung zu verzögern. Gerade für die Bürger als Verbraucher wirkt sich dies zumeist nachteilig aus.

Anja Hampel unterstreicht gleich zu Beginn ihrer Studie, dass die EU-Richtlinie ein wichtiges, wenn nicht sogar eines der zentralen Steuerungsinstrumente der EU sei - eine These, der man insgesamt zustimmen kann, denn die EU-Institutionen verfügen außerhalb ihrer Rechtsetzungs- und der damit verbundenen Sanktionsmöglichkeiten über wenige Instrumente, um die Integrationsentwicklung zu steuern. Zum Teil bleiben Mitgliedstaaten allerdings deutlich hinter den - normierten - Umsetzungsterminen zurück, ein Problem, dessen Ursachen und Gründe Hampel anhand des Vetospieleransatzes analysiert. Ihre daraus abgeleitete Grundthese, dass in erster Linie nationale Vetospielerkonstellationen darüber entscheiden, ob EU-Staaten in der Umsetzung von Richtlinien „Musterknaben“ oder „Nachzügler" sind, ist interessant und originell, wobei die Autorin im Vorfeld ihrer ausführlichen empirischen Analyse andere Erklärungsansätze diskutiert und ablehnt - zwar etwas knapp, jedoch insgesamt zufriedenstellend.

Die als „Fallbeispiele“ gekennzeichnete empirische Untersuchung rekonstruiert die nationalen Implementationsprozesse und Vetospielerkonstellationen am Beispiel der Antidiskriminierungsrichtlinie. Diese Auswahl ist gelungen, da diese Richtlinie, die genau genommen aus zweien besteht, auf nationaler Ebene zum Teil sehr umstritten war und der Leser bereits vor Lektüre des Kapitels ahnt, dass sich die Vetospielerkonstellationen zum Teil polarisiert darstellen. So kommt Hampel nach eingehender Erläuterung der rechtlichen Voraussetzungen und der Vetospielerkonstellationen zu dem Ergebnis, dass gerade in Deutschland die hohe Zahl an relevanten Vetospielern und deren hohe Inkongruenz die Umsetzung der Richtlinie zeitlich zunächst stark verzögerten. Der Vergleich mit Österreich, Irland und Großbritannien scheint die Grundthese der Arbeit ebenfalls zu bestätigen, obgleich es dort zu weniger Verzögerungen kam.

Allerdings ist zu bedauern, dass sich Hampel in ihren Fallbeispielen auf diese vier Länder beschränkt. Der Vergleich insbesondere mit EU-Staaten aus dem südeuropäischen Raum wäre sehr interessant gewesen, da vor allem hier eine empirische Überprüfung der Grundthese Stärken und Schwächen des gewählten Ansatzes unter Umständen noch deutlicher hätte werden lassen. So ist die Implementationssituation etwa in Italien oder Portugal auch deshalb besonders, weil diese Länder in der Vergangenheit unter anderem aufgrund systemimmanenter Selbstblockaden zur stark verspäteten Umsetzung von EU-Richtlinien gerade- 
zu neigten beziehungsweise sich mit juristischen „Tricks“ wie der Umsetzung ganzer Richtlinienpakete durch Exekutivmaßnahmen behalfen. Zumal Hampel unterstreicht, dass die vorhandenen Erklärungsmodelle nicht hinreichend tragen, um die maßgeblichen Faktoren für die unterschiedliche Geschwindigkeit der Richtlinienimplementation zu identifizieren, hätte eine Beleuchtung der Situation in weiteren EU-Staaten den Erkenntnisgewinn ihrer Arbeit deutlich gesteigert.

Zudem bleibt offen, inwiefern die Europäische Kommission selbst - und andere EUInstitutionen wie etwa der Europäische Gerichtshof - sich im Verlauf kritischer Umsetzungsprozesse de facto zu weiteren Vetospielern entwickeln. Hampel deutet eine solche Tendenz in ihrem Fazit an, indem sie feststellt, dass die ausgesprochen rasche Umsetzung der Richtlinie durch die Große Koalition in Deutschland bei unveränderter Vetospielerkonstellation vor allem dadurch motiviert war, dass die EU Sanktionierungsmaßnahmen angedroht hatte. Die Autorin stellt hierzu fest, dass diese Entwicklung von der Vetospielertheorie nicht hinreichend erklärt werden könne und behilft sich damit, diese als externen beziehungsweise "Störfaktor" zu bezeichnen. Dies bleibt unbefriedigend, zumal Hampel in ihrem Gesamtfazit zuzustimmen ist, dass die Sanktionsmöglichkeiten des EU-Rechts auch bei ungünstigen nationalen Vetospielerkonstellationen letztlich eine Implementation von Richtlinien erzwingen, allerdings mit teilweise enormen zeitlichen Verzögerungen. Dies drängt die Frage auf, inwiefern vor allem die EU-Kommission, die diesen Druck erst erzeugen und sich der Sanktionsinstrumente des EU-Rechts bedienen kann, de facto zu einem Vetospieler wird, der mächtig oder sogar übermächtig auf nationaler Ebene agiert und dessen Einfluss - wie gerade Hampels Fallbeispiel Deutschlands zeigen könnte - den aller übrigen nationalen Vetospieler überflügelt. Zwar gelten die bekannten Einschränkungen des EU-Rechts, wonach rechtliche Sanktionen erst nach dem Umsetzungszeitraum angedroht werden können, während inhaltlich nur eine Mindestannahme der Vorgaben (1:1-Umsetzung) verlangt werden darf. Sind diese Voraussetzungen jedoch erfüllt, wäre zu prüfen, ob die EU-Kommission eine neue Rolle als zentraler Akteur oder gar Vetospieler einnimmt, der zumindest zwei Optionen unmöglich macht: die Nichtumsetzung einer Richtlinie oder deren Umsetzung hinter den geforderten Mindeststandards.

Insgesamt ist das Fazit der Arbeit aufgrund der Fallstudien durchaus überzeugend. Lobend ist hervorzuheben, dass Hampel selbst die Grenzen und eine mögliche Entwicklung der Vetospielertheorie diskutiert. Der Leser wünscht sich dennoch nach der Lektüre eine weitergehende Analyse unter Einbezug anderer EU-Staaten und der EU-Institutionen. Daraus würde sich unter Umständen ein auch theoretisch noch breiter gefächertes Bild ergeben.

Cordula A. Janowski

\section{Jahre V. Republik Frankreichs}

Deutsch-Französisches Institut (Hrsg.): Frankreich Jahrbuch 2007. 50 Jahre V. Republik, VS Verlag für Sozialwissenschaften, Wiesbaden 2008, 324 Seiten, € 44,90.

Zum 50. Jahrestag des Bestehens der V. Republik erschienen, wählt das Frankreich Jahrbuch natürlicherweise ihr politisches System zum Schwerpunkt. Insgesamt zwölf Beiträge (acht von französischen Autoren, drei von deutschen, einer von einem Engländer), hervor- 